\title{
El Software de oficina y el estudiante universitario
}

\author{
Office software and the university student
}

Emilio Alejandro Rivera-Landero ${ }^{\text {a }}$

\begin{abstract}
:
At present, is necessary to ensure that students and graduates of the higher level have the necessary digital skills that allow them to function properly in the professional world and not be considered digital illiterates; among these competencies, the work with office software is essential. In this idea, Higher Education Institutions have a very important role to promote the digital skills, performing this work explicitly with subjects that are focused on the technical learning of office and / or implicit way, where from each subject the teacher promotes work with the required software.
\end{abstract}

Keywords:

Office software, digital skills

\section{Resumen:}

En la actualidad, es necesario asegurar que los alumnos y egresados del nivel superior cuenten con las competencias digitales necesarias que les permitan desenvolverse adecuadamente en el mundo profesional y no ser considerados analfabetas digitales; de entre estas competencias resulta básico el dominio del software de oficina. En este sentido, las Instituciones de educación superior tienen un papel de suma importancia para fomentar el dominio de dichas competencias, realizando esta labor de forma explícita con materias que estén enfocadas al aprendizaje técnico del software de oficina y/o implícita, donde desde cada materia el docente promueve el dominio del software requerido.

\section{Palabras Clave:}

Software de oficina, ofimática, competencias digitales

\section{Introducción}

El desarrollo de las competencias digitales en los alumnos y específicamente en aquellos de nivel superior es un tema que se ha abordado en diversos foros tanto presenciales como digitales por especialistas en la materia, en dichos espacios la importancia de que los futuros profesionistas las dominen es algo que encuentra una adhesión prácticamente unánime.

Este trabajo se une a tales voces clarificando algunos elementos que las componen para entender mejor a qué nos referimos con las competencias digitales, haciendo énfasis en el estudio y manejo de programas de oficina.

Por ello, en el presente ensayo, se presentan en un primer lugar las nociones básicas para entender qué es una competencia digital, cuál es su utilidad, cómo esta enlazada con la informática y la cultura digital, para evitar confundirla con la simple convivencia diaria e incluso fuera de límites recomendados con la tecnología, que puede llevar a la tecnofilia.

Una vez abordadas las nociones básicas se tratan con mayor detalle los elementos que componen las competencias digitales desde de la propuesta desarrollada por Ramírez y Casillas (2017) consistente en un esquema construido a partir de las coincidencias entre marcos de referencias de la OCDE (Organización para la Cooperación y el Desarrollo Económicos) la UNESCO (La Organización de las Naciones Unidas para la Educación, la Ciencia y la Cultura) la ECDL (European Computer Driving Licence) y la ISTE (Sociedad Internacional de Tecnología en Educación).

Posterior al establecimiento de este marco de referencia se hace énfasis en el lugar que dentro del universo de las competencias digitales, ocupa el dominio del software de oficina y la importancia de abordarlo en el nivel superior

Autor de Correspondencia, Universidad Autónoma del Estado de Hidalgo, https://orcid.org/0000-0002-8685-3746, Email: rlandero@uaeh.edu.mx 
tanto de forma explícita como implícita a lo largo de la formación del estudiante universitario.

Finalmente, se presentan las conclusiones fruto de la investigación realizada y de un ejercicio de reflexión personal desde la práctica de la docencia.

\section{Desarrollo}

De acuerdo a Díaz, Cebrián y Fuster (2016) las competencias digitales son conocimientos y habilidades sobre recursos tecnológicos que permiten, según lo establecido por Zuñiga, Edel y Lau (2016) llevar la interaccióntecnológica a campos de desarrollo académico de forma segura y crítica.

Es posible ya desde esta primera definición vislumbrar algunos elementos básicos de las competencias digitales, especialmente en alumnos de nivel superior, entre estos elementos se distinguen: la complementariedad entre los conocimientos y las habilidades, no basta con saber hacerlo, es necesario mostrar el dominio dentro de un contexto que aporte elementos de mejora desde el punto de vista académico o intelectual y aun más, se ponen en juego aspectos éticos.

Las competencias digitales por tanto no se limitan al simple hecho de saber utilizar la tecnología, Ferreiro (2009) citado por Zuñiga, Edel y Lau (2016) declara que la adicción tecnológica (tecnofilia) del ciudadano de la segunda década del presente siglo, no representa necesariamente competencia en materia de gestión del conocimiento en el entorno virtual.

Es de resaltar que la tecnofilia es una adicción donde la tecnología es parte de la vida diaria, sin embargo no se le da una aplicación ideal provocando en quien la padece una dependencia, siendo absorbidos por la tecnología su tiempo y recursos tanto económicos como incluso por llamarlos así, emocionales.

Por tanto es posible establecer que un alumno universitario que ha convivido con la tecnología y hace uso de, por ejemplo, un teléfono celular o una consola de videojuegos o de redes sociales; no es necesariamente un alumno competente en los aspectos digitales, ya que puede carecer de los elementos antes citados respecto de su aplicación en áreas académicas así como de realizarlo de forma segura y crítica.

Siguiendo esta línea de análisis, el correcto dominio de las competencias digitales debe permitir a un alumno utilizar la informática para el procesamiento automático de la información mediante los dispositivos y sistemas que su área de especialidad requiera así como aquellos que son aplicables a todas las áreas del conocimiento tal como el Software de oficina, el cual se comenta más adelante. Dicho dominio también le permitirá al alumno formar parte de una cultura tecnológica que le de soporte a su formación profesional con miras a su desarrollo laboral o de investigación en aspectos como la adquisición de conocimiento, la comunicación, el trabajo en equipo e individual así como el fomento de su creatividad (Rosado y Espinoza, 2015)

Una vez establecidos los elementos básicos para comprender las competencias digitales, es oportuno clarificar los saberes digitales que las conforman. Se entienden los saberes digitales como:

"una estructura graduada de habilidades instrumentales y conocimientos teóricos de carácter informático e informacional que distinguen a los usuarios de las Tecnologías de la Información y la Comunicación (TIC) conforme al contexto académico en el que se desenvuelven." Ramírez y Casillas (2017)

Los mismos autores proponen un esquema de saberes digitales a partir de las coincidencias entre los marcos de referencia de la OCDE, la UNESCO, la ECDL y la ISTE, estru ctu rada de forma general en los siguientes saberes y que si bien está pensada en la formación de docentes puede ser adaptada a cualquier profesional: usar dispositivos, administrar archivos, usar programas y los sistemas de información especializados, creary manipular contenido de texto y texto enriquecido, crear y manipular conjuntos de datos, crear y manipular medios y multimedia, comunicarse en entornos digitales, socializar y colaborar en entornos digitales, ejercer y respetar una ciudadanía digital, y finalmente, literacidad digital.

De entre dichos saberes resalta el uso de los programas de oficina es decir la creación y manipulación de textos, presentaciones electrónicas y, manejo de datos en forma de hojas de cálculo. Lo anterior, ya que su dominio representa algo esperado y requerido por el mercado laboral debido a la alta competitividad que hoy demanda la sociedad de la información. Para apoyar esta tarea existe un amplio abanico de posibilidades en cuanto al software a utilizar, por ejemplo: programas en la nube como las herramientas en línea de Google, software libre como WPS o bien Suites de oficina de paga como Microsoft Office; cada una de las cuales cuenta con programas de Procesamiento de textos, Hojas de cálculo electrónicas y Presentaciones electrónica; los cuales pueden ser manipulados en un equipo de cómputo de oficina o bien en una tableta o un teléfono inteligente.

Estos saberes si bien son desarrollados en algunos casos desde la formación básica, es posible observar que una gran parte de los estudiantes de nivel superior no cuentan con un manejo aceptable que les permita iniciar su preparación académica y quienes tienen un dominio técnico básico de estas herramientas no logran distinguir el aspecto positivo de dichos saberes en cuanto a tres áreas que mencionan Rosado y Espinoza (2015): la mejora en la planificación de tareas, la resolución de problemas y la transmisión de nuevos conocimientos. 
Los docentes y los centros educativos no pueden dar por dominado o bien minimizado el manejo del software de oficina por parte de los alumnos, dado que en una gran parte de los talleres, empresas y organizaciones que contratan al profesional que egresa de nuestras escuelas, lo consideran como un aspecto requerido para poder ingresar en la vida laboral y que les permite llevar a cabo tareas que pueden ir desde la creación de nuevos formatos hasta presentar proyectos, ofrecer reportes, realizar análisis de datos y otras con mayor especialización.

Por lo anterior es importante que en la Universidad se desarrollen adecuadamente estos saberes. Ante esta situación existen dos vertientes: la de implementar materias que de forma específica los aborden y la que establece, que no es necesario este tipo de materias ya que lo mejor es desarrollar estos saberes en todas las materias de acuerdo a los objetivos de cada una; en ambos casos es necesario buscar que el desarrollo de tales saberes sea desde estrategias y paradigmas que busquen reducir la brecha digital que hoy existe entre alumnos de nivel superior.

Estas dos vertientes tienen ventajas y desventajas, por ejemplo, mientras que al implementar materias específicas para el desarrollo de habilidades con software oficina se asegura que hay un espacio dentro del cual se fomentará, es posible que la relación con el software se presente solucionando problemas que no tengan vinculación con la disciplina de estudio del Programa educativo.

Por otra parte, al optar por el desarrollo del dominio de software de oficina desde cada materia del Programa educativo, se logra que los problemas a solucionar estén contextualizados dentro del tipo de soluciones que ofrece la disciplina, sin embargo puede suceder que el docente de cada materia no los utilice, prefiriendo el uso de otros programas dejando al estudiante sin el dominio de los saberes digitales enfocados al manejo de software de oficina.

En ambos casos, optando por una u otra estrategia se requiere de coordinación entre las materias que conforman el programa educativo con el objetivo de asegurar el desarrollo de los saberes que conforman el dominio de un software de oficina, insistiendo que estos son parte de las competencias digitales necesarias y esperadas en todo estudiante y egresado. En este sentido y si bien no es materia del presente ensayo, para lograr una adecuada coordinación y objetivos comunes, es notoria la importancia del trabajo de las academias, en donde además se gestarán los paradigmas citados en párrafos anteriores.

Estos nuevos paradigmas deben considerar entre otros aspectos, las evidencias que muestran que ante mayor frecuencia de uso de un software se obtiene mayor dominio del saber (Díaz, Cebrián y Fuster, 2016). Por lo que el desarrollo debe ser constante y utilizando todos los medios que hoy se encuentran al alcance de los docentes y alumnos.

\section{Conclusiones}

Para concluir, el manejo de software de oficina se encuentra inserto dentro de los saberes que un profesional debe tener como parte de sus competencias digitales y es de suma importancia que desde la Universidad se busque el dominio de tales saberes en un nivel avanzado por parte de los alumnos.

Es necesario que el alumno vea a su tableta o teléfono inteligente como una herramienta más de su formación, instalando en el dispositivo el software de oficina preferido, de tal manera que no se requiera situarse en una sala de cómputo para hacer uso de los programas de oficina.

Finalmente, si bien existen dos maneras de abordar dicho dominio, ambas con ventajas y desventajas, desde una experiencia personal como docente, es posible mencionar que lo importante es el compromiso de cada docente por promover el uso de la tecnología y en especial de los programas de oficina así como la coordinación entre materias y desde las academias; descubriendo su potencial en la solución de problemas propios de la disciplina en que se desenvuelva o desenvolverá el alumno. Este compromiso para con la formación del alumno ayudará a que el uso del software sea constante y parte sustancial de ambientes de aprendizaje donde se promueva el aprendizaje significativo.

\section{Referencias}

[1] Díaz García Isabel, Cebrián Cifuentes Sara, Fuster palacios Isabel (2016). Las competencias en TIC de estudiantes universitarios del ámbito de la educación y su relación con las estrategias de aprendizaje. RELIEVE, 22 (1), art. $5 . \quad$ DOI: http://dx.doi.org/0.7203/relieve.22.1.8159

[2] Ramírez Martinell Alberto, Casillas Miguel Ángel (Coordinadores) (2017) Saberes digitales de los docentes de educación básica. 1a Edición, SEP Veracruz México.

[3] Rosa do Álvarez María, Espinoza Burgos Álvaro (2015) "Importancia de la informática en prácticas profesionales o pre-profesionales de estudiantes de pregrado" en Atlante. Cuadernos de Educación y Desarrollo, febrero 2015, Recuperado de http://atlante.eumed.net/importancia-informatica/

[4] Cosas de educación (2016) La importancia de la ofimática, Recuperado de https://www.cosasdeeducacion.es/la-importancia-de-la-ofimatica. 5 de diciembre de 2019.

[5] Zuñiga Lobato José Iram, Edel Navarro Rubén, Lau Noriega Jesús. (2016) Competencias digitales y educación superior. Revista de transformación educativa. Recuperado de https://rete.mx/attachments/article/7/RETE\%20- 
Publicación semestral, Ingenio y Conciencia Boletín Científico de la Escuela Superior Ciudad Sahagún, Vol. 7, No. 14 (2020) 62-64

\%20Competencias\%20digitales\%20y\%20educaci\%C3\%B3n\%20super ior.pdf 5 de diciembre de 2019 . 